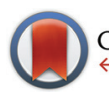

CrossMark \&lick for updates

Cite this: Dalton Trans., 2014, 43 17395

Received 2nd October 2014 Accepted 10th October 2014 DOI: $10.1039 / c 4 d t 03048 f$ www.rsc.org/dalton

\title{
Rational design of a room temperature molecular spin switch. The light-driven coordination induced spin state switch (LD-CISSS) approach $\dagger$
}

\author{
M. Dommaschk, ${ }^{a}$ C. Schütt, ${ }^{a}$ S. Venkataramani, ${ }^{b}$ U. Jana, ${ }^{c}$ C. Näther, ${ }^{d}$ \\ F. D. Sönnichsen ${ }^{a}$ and R. Herges ${ }^{\star a}$
}

\begin{abstract}
Extensive use of quantum chemical calculations has been made to rationally design a molecule whose spin state can be switched reversibly using light of two different wavelengths at room temperature in solution. Spin change is induced by changing the coordination number of a nickel complex. The coordination number in turn is switched using a photochromic ligand that binds in one configuration and dissociates in the other. We demonstrate that successful design relies on a precise geometry fit and delicate electronic tuning. Our designer complex exhibits an extremely high long-term switching stability (more than 20000 cycles) and a high switching efficiency. The high-spin state is extraordinarily stable with a half-life of 400 days at room temperature. Switching between the dia- and paramagnetic state is achieved with visible light (500 and $430 \mathrm{~nm}$ ). The compound can also be used as a molecular logic gate with light and $\mathrm{pH}$ as input and the magnetic state as non-destructive read-out.
\end{abstract}

\section{Introduction}

Bistability of spin states has long been restricted to solid state systems or very low temperatures. Spin state switching in isolated molecules or in homogenous solution ${ }^{1}$ at room temperature, however, is still a challenge. ${ }^{2-4}$ A number of interesting applications are waiting to be exploited for these systems, such as switchable contrast agents for MRI, ${ }^{5,6}$ light-controlled magnetic levitation, switchable spin labels, ${ }^{7}$ or spintronics. ${ }^{8,9}$ Unsurprisingly, a number of groups are aiming at this end using different approaches. Starting point of the LD-LISC (light-driven ligand-induced spin change) approach is the spin crossover of $\mathrm{Fe}^{2+}$ as a function of the ligand field strength. Photochromic compounds are used as light switchable ligands. The two configurations of the photochromic ligands exhibit different ligand field strengths ${ }^{10,11}$ which eventually give rise to different spin states. ${ }^{12}$ Redox processes can be used

${ }^{a}$ Otto-Diels-Institut für Organische Chemie, Christian-Albrechts-Universität, Otto-Hahn-Platz 4, D-24098 Kiel, Germany.E-mail: rherges@oc.uni-kiel.de

${ }^{b}$ Indian Institute for Science Education and Research (IISER) Mohali, Knowledge City, Sector 81, SAS Nagar, Manauli PO 140306, India

${ }^{c}$ Department of Chemistry, Jadavpur University, Kolkata-700032, India

${ }^{d}$ Institut für Anorganische Chemie, Christian-Albrechts-Universität, Max-Eyth-Straße 2, 24098 Kiel, Germany

$\dagger$ Electronic supplementary information (ESI) available. CCDC 1023485 For ESI and crystallographic data in CIF or other electronic format see DOI: $10.1039 / \mathrm{c} 4 \mathrm{dt} 03048 \mathrm{f}$ to change the spin state of transition metals. ${ }^{13}$ Photo switchable antiferromagnetic coupling was demonstrated in nitroxide substituted diarylethenes. ${ }^{9,14}$ A magnetic switch from paramagnetic to ferromagnetic has been achieved by moving two Co-phthalocyanine molecules $(S=1 / 2)$ on top of each other $(S=1)$ with a STM tip. ${ }^{15}$ Reversible spin-crossover was also observed by electron injection in single molecules on a surface with an STM tip. ${ }^{16,17}$

Recently we presented the first molecular spin switch that can be operated by visible light at room temperature in homogenous solution with no measurable fatigue over more than 20000 cycles. ${ }^{18}$ Our approach is based on the well-known fact that a number of transition metal ions, such as $\mathrm{Fe}^{2+}, \mathrm{Fe}^{3+}$, $\mathrm{Mn}^{2+}, \mathrm{Mn}^{3+}, \mathrm{Co}^{2+}$, and $\mathrm{Ni}^{2+}$ change their spin state upon changing the coordination number. $\mathrm{Ni}^{2+}$ was chosen as the transition metal for several reasons: 1 . There is a reliable spin state switch between low-spin $(S=0)$ and high-spin $(S=1)$ if the coordination is changed from square planar (coordination number $\mathrm{CN}=4)$ to square pyramidal $(\mathrm{CN}=5)$ or square bipyramidal $(\mathrm{CN}=6) \cdot{ }^{19-21}$ (see Fig. 1) $2 . \mathrm{Ni}^{2+}$ high-spin complexes still exhibit reasonably sharp NMR spectra for analysis, and paramagnetic shifts can be used to determine the ratio of high-spin/low-spin $\mathrm{Ni}$ ions in solution ${ }^{22-24} 3 . \mathrm{Ni}^{2+}$ complexes are easier to calculate than $\mathrm{Fe}^{2+}$ compounds (more reliable convergence of the wavefunction to the lowest electronic state). In the first place we accepted the disadvantage that the highspin state of $\mathrm{Ni}^{2+}(S=1)$ has a lower magnetic moment than 

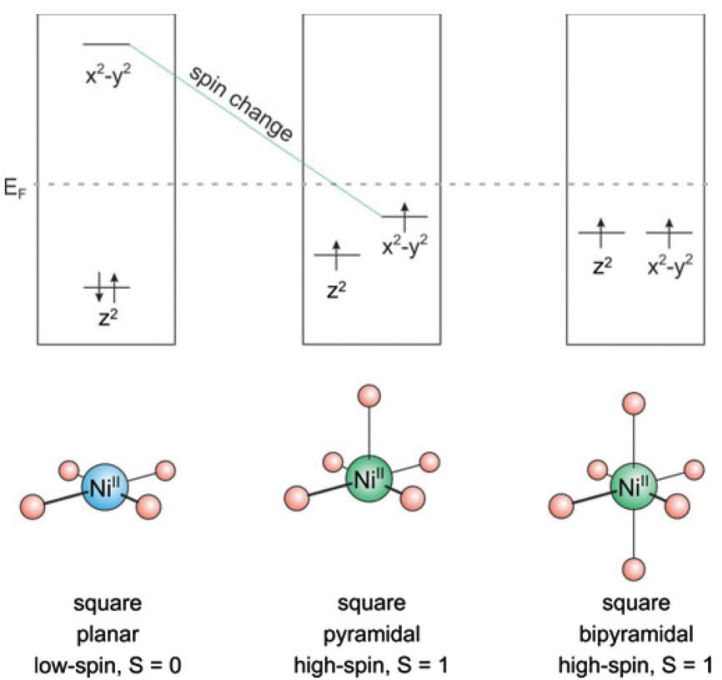

Fig. 1 Schematic illustration of coordination induced spin state switch in $\mathrm{Ni}$-porphyrin.

$\mathrm{Fe}^{2+}(S=2)$. We chose Ni-porphyrin as a square planar complex $(\mathrm{CN}=4)$ and an azopyridine tethered to the porphyrin as the photoswitch and light-controlled axial ligand.

We now present a detailed analysis of the prerequisites of the design and optimization of spin switches based on this Light-Driven Coordination Induced Spin State Switch (LD-CISSS) approach. The optimal system is completely diamagnetic (low-spin) in one state and completely paramagnetic (high-spin) in the other state. However, 100\% switching efficiency is difficult to achieve in an ensemble of individual molecules. The overall magnetic switching efficiency depends on the (cis-trans) switching efficiency of the photochromic ligand and on the association constants in both configurations of the ligand. The binding constant should ideally be zero in one state and large in the other. Unfortunately, the situation becomes considerably more complicated under "real" conditions where solvent effects and intermolecular binding interfere. The solvent itself can bind as an axial ligand and convert the nickel complex to the high-spin state, even in the nonbinding state of the photochromic ligand. At a first glance, a high binding constant of the photochromic ligand in the binding state should be desirable. However, two problems arise if the association constant is too large. Switching to the non-binding state is thermodynamically less favourable and could be impaired. Moreover, favouring intramolecular binding would also favour intermolecular association. At increasing concentrations an increasing proportion of the complex would form dimers or oligomers and would always be high-spin. Further complications arise from the fact that nickel-porphyrins can add a second axial ligand to form a distorted octahedral complex (Fig. 1). Addition of the first ligand (e.g. photochromic ligand) changes spin state from low- to high-spin and activates the addition of a second axial ligand $\left(K_{2}\right.$ usually is larger than $K_{1}$ ). Weakly coordinating solvents that do not bind to the square planar Ni porphyrin could still bind to the square pyramidal complex (binding state of the photochromic ligand) and stabilize the high-spin state which would increase the switching efficiency. Another issue is the life time of the thermodynamically less stable state (usually the cis isomer in azobenzenes) which should be large in an ideal system. Many photochromic systems including azobenzenes and spiropyrans undergo thermal back reaction to the more stable state. Coordination could be used to stabilize the binding state of the photochromic ligand if the less stable configuration is binding. A positive feedback from coordination could even improve the switching efficiency of the photochromic ligand. On the other hand the switching of the photochromic ligand often is completely quenched if a chromophore such as a porphyrin is in close proximity or in conjugation. This has to be considered in the design of the tether that connects the porphyrin and the photochromic ligand.

Hence, a number of preconditions have to meet to design molecular spin switches based on the LD-CISSS approach: perfect geometric and electronic design of the switching ligand including the tether, a delicate tuning of the electronic properties of the Ni porphyrin and of the donor strength of the photochromic ligand, the choice of the solvent etc. Detailed information about these parameters is of utmost importance for the design of optimized molecular spin switches for various applications such as contrast agents for MRI or switchable spin labels.

We here report on detailed investigations of the above parameters using NMR and UV-vis spectroscopy and single crystal structure analysis. To elucidate the mechanism of the spin switch we performed quantum chemical calculations and compare experimental and theoretical data of two Ni porphyrins (Ni-TPPF 20 and Ni-TPP).

\subsection{General design}

The trivial way to change the coordination number of Ni-porphyrin would be to add a ligand to the solution. This would lead to a continuous increase of the higher coordinated complex but not to a switching process, and it would not be reversible. We chose light as the trigger, because it can be applied in different wavelengths for switching back and forth with temporal and spatial control, and unlike chemical triggers light does not leave a trace if it is switched off. So initially we have to design ligands that can be switched between two states: a binding and non-binding form using light of two different wavelengths. To keep the design as simple as possible, we chose 3-phenyl-azopyridine as the photochromic ligand because it combines the switching properties of azobenzene and the coordination of pyridine in a single small molecule. 2-Phenyl-azopyridine and 4-phenyl-azopyridine are not suitable because the first doesn't coordinate to square planar metal complexes (sterical hindrance) and the latter binds in both configurations. Hence, the building blocks to work with are Ni-porphyrin and 3-phenyl-azopyridine. Both units have to be connected with each other by means of a tether in such a way that the lone pair of the pyridine nitrogen is exactly orthogonal to the porphyrin plane in a distance of about $2.2 \AA$. 
a)

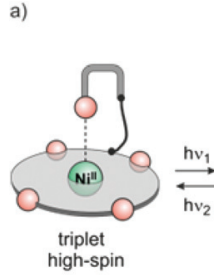

b)

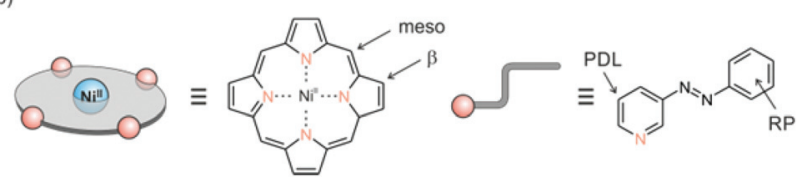

Fig. 2 (a) General design approaches (record player (RP), left), photodissociable ligand (PDL) right. (b) Positions of tether attachment.

There are two points where the tether could be attached to the porphyrin (meso or $\beta$ ). The other end of the tether can be connected to either the pyridine (PDL design) or the phenyl ring (RP design) of the 3-phenyl-azopyridine unit (Fig. 2). The choice of the position of attachment at the phenyl-azopyridine is of particular importance. A number of azobenzene, azoporphyrin or stilbazole substituted porphyrins are known. However, none of the molecules with ortho or para connection to the porphyrin exhibits photochromic behavior. ${ }^{25-29}$ Conjugation to a chromophore with a low lying $\mathrm{S}_{1}$ state probably quenches the $\pi-\pi^{*}$ excitation of the azo group and thus prevents photochemical isomerisation. ${ }^{30}$ Therefore, a direct conjugation path between the azo group and the porphyrin has to be avoided either by an $\mathrm{sp}^{3}$ atom or a meta connection within the tether. Attachment at the ortho position of the pyridine ring would prevent coordination by steric hindrance. So we are left with several combinations for attachment and a plethora of conceivable tethers with different lengths and geometries (Fig. 2).

\section{Results}

\subsection{Computational modeling}

At this point we used molecular construction kits to build models and performed density functional theory calculations (B3LYP/6-31G*) on more than 36 different structures that looked promising based on geometry and synthesizability. (a selection of structures is presented in ESI $\dagger$ ) For obvious reasons the structures were coined "record players" because the porphyrin resembles a disk, the azopyridine works like a tone arm by placing the pyridine nitrogen lone pair (needle) onto the nickel ion.

The structures were ranked according to their theoretically predicted performance. Target parameters are the optimal distance of the pyridine nitrogen and the nickel atom in the binding configuration and a minimal deviation from orthogonal binding to the porphyrin plane. The optimal N-Ni bond length was determined by calculations of phenyl-azopyridine porphyrin complexes without a tether and complete optimisation of all geometry parameters including the $\mathrm{N}-\mathrm{Ni}$ distance
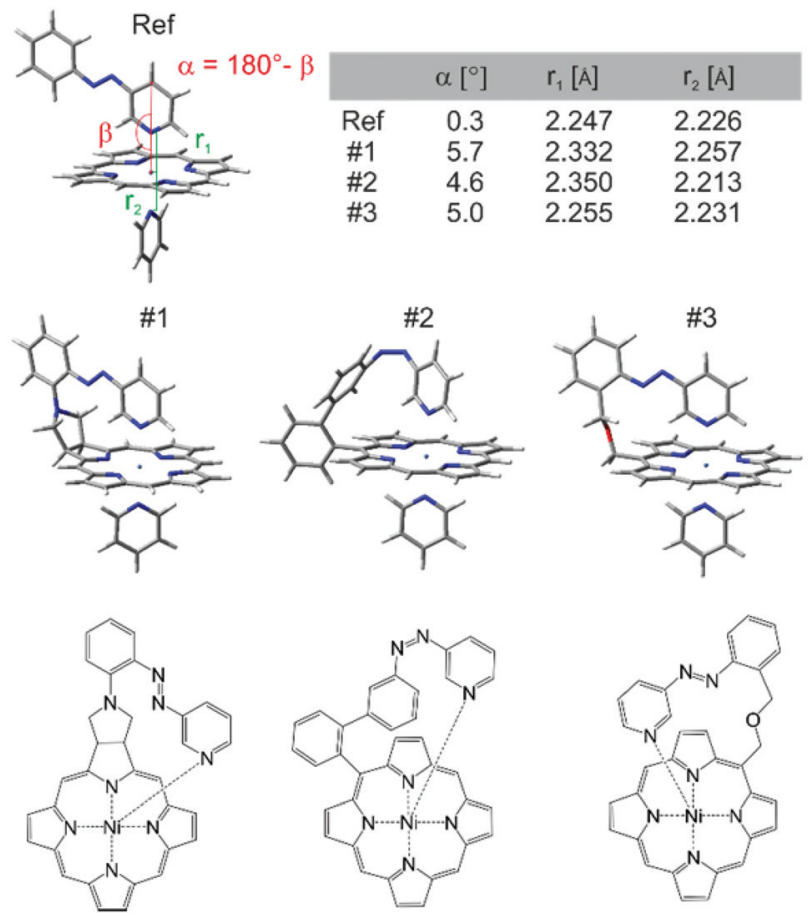

Fig. 3 Geometry plots (B3LYP/6-31G*) and structures of Ni-porphyrin with 3-phenyl-azopyridine and pyridine as axial ligands. The reference compound (Ref) without tether is given on top left, and the three highest ranking "record player" structures for LD-CISSS are represented underneath. Note that structure \#1 and \#3 bind in trans configuration and \#2 coordinates in its cis form. Pyridine has been included in the calculations as a second axial ligand because it is known that $\mathrm{Ni}$-porphyrins prefer a 6-coordinate ligand field. It is assumed that upon changing the coordination number from 4 to 5 and concomitant switching from lowspin to high-spin a solvent molecule would coordinate to complete the square bipyramidal ligand field.

(2.075 A without and $2.247 \AA$ with pyridine as the sixth ligand, see structure denoted as Ref. in Fig. 3,). Three candidates turned out to comply very well with the geometry constraints above (\#1, \#2, and \#3, Fig. 3).

"Record player" structure \#1 is predicted to be high-spin (binding) in its trans configuration and low-spin in its cis form. A distinct advantage over structures \#2 and \#3 is the reduced conformational flexibility which should lead to a better switching efficiency. Unfortunately all attempts to synthesize structure \#1 failed. The 1,3-dipolar cycloaddition of azomethine ylides to the pyrrole double bonds of porphyrins is known. ${ }^{31-33}$ Cross coupling of the pyrrolidine nitrogen with 3-(2-iodo-phenylazo)-pyridine, however, resulted in coupling with the pyridine ring. Several other synthetic attempts failed as well. We therefore discontinued the synthesis in favor of record player \#2.

The synthesis of structure \#2 with phenyl groups in the porphyrin meso positions via the mixed aldehyde method was straightforward. ${ }^{33}$ Unfortunately, irradiation with light of $365 \mathrm{~nm}$ which usually leads to efficient trans-cis isomerisation in azobenzenes and azopyridines was incomplete (32\% cis). Photo isomerisation of the cis form back to trans with $420 \mathrm{~nm}$ 


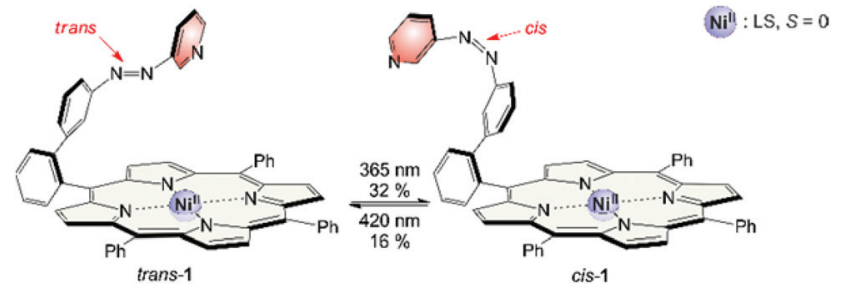

Fig. 4 Record player \#2 with phenyl groups in meso position (1) does not switch efficiently upon irradiation. The photostationary state (PSS) upon irradiation at $365 \mathrm{~nm}$ is $32 \%$ cis and at $420 \mathrm{~nm} 16 \%$ cis. Even though the geometry of the cis isomer is optimal for coordination, the "tone arm" doesn't bind to the $\mathrm{Ni}^{2+}$.

was incomplete as well (16\% cis left). Back-isomerisation to the pure trans isomer was achieved by heating to $70{ }^{\circ} \mathrm{C}$ for several hours. Furthermore the cis isomer does not exhibit paramagnetic behavior as expected. Only $5 \%$ of the molecules are in the triplet state. Instead of binding to the $\mathrm{Ni}^{2+}$, the azopyridine "tone arm" rotates away from the porphyrin. This hypothesis was corroborated by UHV STM measurements at $5 \mathrm{~K}^{33}$ The molecules were deposited on an $\mathrm{Au}(111)$ surface and manipulation of the azopyridine unit was achieved by the STM tip. The trans-cis isomerisation is clearly visible in the STM image. The azopyridine arm points straight away from the porphyrin core and it is bent in cis configuration (Fig. 4).

To elucidate the reason for this almost complete failure, we performed further DFT calculations with emphasis on the binding energy of pyridines as axial ligands, and on the singlet-triplet gap of Ni-porphyrins with different substituents in meso position. Among 10 different functionals in combination with 5 different basis sets B3LYP/def2TZVP performed best $^{34}$ in predicting our experimentally determined association energies including the formation of $1: 1$ and $2: 1$ complexes of porphyrins with pyridines. Simple PBE/DZP seems to be sufficient for geometry optimisation as was confirmed by comparison with our X-ray structures, and by comparing B3LYP/ def2TZVP single point association energies at geometries obtained by optimisation with different functionals and basis sets (for computational details see ESI†). In Table 1 and Fig. 5 the corresponding data for Ni-tetraphenyl-porphyrin (Ni-TPP) and Ni-tetrakis(pentafluoro-phenyl)-porphyrin $\left(\mathrm{Ni}-\mathrm{TPPF}_{20}\right)$ (the latter as an example for an electron poor porphyrin) are presented. The calculated values are compared with experimental association energies that were obtained by NMR titration at different temperatures. ${ }^{22}$ (Table 1, Fig. 5). It is important to note that neither of the Ni-porphyrins binds pyridine as an axial ligand in its singlet spin state. ${ }^{35}$ Our calculations reveal a strong repulsion between the $\mathrm{Ni}$ ion and the pyridine nitrogen lone pair. Upon including dispersion energy (D3) ${ }^{36}$ sandwich type structures were found, however, with no coordination between nickel and the pyridine nitrogen. One can assume that these van der Waals complexes do not exist in homogenous solution due to competition of the pyridine with the solvent. Therefore we conclude that there is no axial coordination in the singlet state at all.
Table 1 Calculated (B3LYP/def2TZVP//PBE/DZP) relative energies (kcal $\mathrm{mol}^{-1}$ ) of the triplet porphyrins Ni-TPP and Ni-TPPF 20 as a function of the number of bound pyridines. The energies are relative to the corresponding singlet state. All energies include two pyridine molecules (and thus are based on the same stoichiometry). The number of bound pyridines is given in the headline. So the energies can be directly compared and interpreted as binding energies. The experimental value for $\Delta H$ of $\mathrm{Ni}-\mathrm{TPP}(\mathrm{ESI})$ and Ni-TPPF 20 are included as well ${ }^{22}$

\begin{tabular}{llll}
\hline Porphyrin (triplet state) & 0 Py $^{a}$ & 1 Py $^{b}$ & 2 Py \\
\hline Ni-TPP calc. & 12.04 & -1.96 & -4.17 \\
Ni-TPP exp. $^{c}$ & - & - & $-4.6( \pm 0.2)$ \\
Ni-TPPF $_{20}$ calc. $^{22}$ & 10.59 & -6.96 & -11.67 \\
Ni-TPPF $_{20}$ exp. $^{2}$ & - & $-5.3( \pm 0.1)$ & $-11.2( \pm 0.5)$
\end{tabular}

${ }^{a}$ Relative energy includes two uncoordinated pyridine molecules.

${ }^{b}$ Relative energy includes one uncoordinated pyridine. ${ }^{c}$ Methods for the determination of the association constant see ESI.

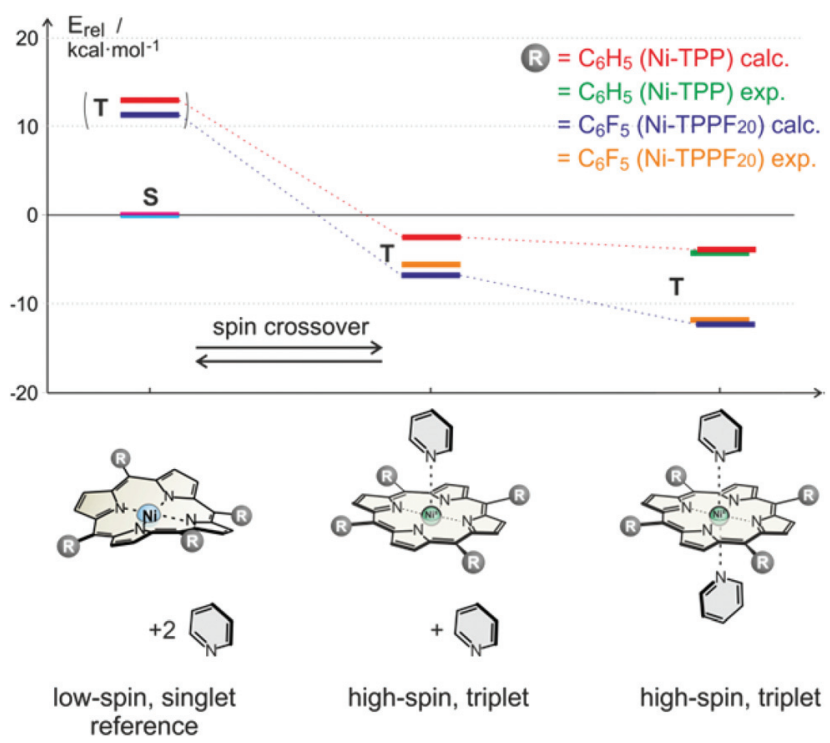

Fig. 5 Calculated (B3LYP/def2TZVP//PBE/DZP) and experimental relative association energies ( $\mathrm{kcal} \mathrm{mol}^{-1}$ ) of pyridine to Ni-TPP (calc. red,

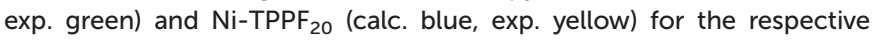
triplet (high-spin) complexes. The energies are relative to the corresponding porphyrin in its low-spin singlet state (including two unbound pyridine molecules). Note that the experimental ${ }^{22}$ and calculated association energies agree quite well. The (very small) experimental binding enthalpy for the 1:1 complex of the Ni-tetraphenylporphyrin with pyridine could not be experimentally determined.

Electron withdrawing substituents in meso position of $\mathrm{Ni}$ porphyrins increase the binding energy of axial ligands by lowering the $d_{z^{2}}$ orbital. It has been shown that the association constants of piperidine as axial ligands to Ni-porphyrins with a number of different para substituted phenyl groups at meso position follow a Hammett relationship. The binding constant ( $2: 1$ complex) of the meso-tetrakis-(4-nitrophenyl) derivative is more than 10 times higher than the association constant of the parent Ni-tetraphenyl-porphyrin. ${ }^{37} \mathrm{~A}$ reverse electronic effect is observed for axial ligands. Electron donor substituents in 4-position of pyridine increase the binding energies to 
Ni-porphyrin also following an approximate Hammett (or basicity) relationship. ${ }^{22,38}$ Our calculations confirm the increase in binding strength of pyridine as a function of electron withdrawing substituents in meso-position of the porphyrin. The calculated association energy of pyridine to Ni-TPPF 20 $\left(-6.96 \mathrm{kcal} \mathrm{mol}^{-1}\right)$ is 3.6 times higher than to the Ni-TPP $\left(-1.96 \mathrm{kcal} \mathrm{mol}^{-1}\right)$. This effect can be easily rationalized using qualitative MO theory. Triplet (high-spin) Ni-porphyrin has an unpaired electron in the $d_{z^{2}}$ as well as in the $d_{x^{2}-y^{2}}$ orbital. The $\mathrm{d}_{z^{2}}$ orbital is mainly responsible for the binding strength of axial ligands. Electron withdrawing substituents at the porphyrin meso-position lower the energy of the $\mathrm{d}_{z^{2}}$ orbital and increase binding. Concomitant with a higher binding energy is a larger singlet-triplet gap in the triplet state. Hence the propensity to undergo spin state change from singlet (low-spin) to triplet (high-spin) should increase with increasing electron withdrawing power of substituents in meso position of the porphyrin ring. An analogous effect should also apply for the record player design. This is why we set out to synthesize record player \#2 with electron withdrawing pentafluorophenyl substituents (2) in the three available meso positions. The syntheses and a rough characterisation were presented previously. ${ }^{18}$

\subsection{Photochromism}

Record player 2 shows an unexpected photochromic behavior. We found that the highest conversion to the cis isomer (75\%) is achieved by irradiation into the porphyrin $\mathrm{Q}$ band at around 495-530 nm. By irradiation into the Soret band of the cis isomer at 420-435 $\mathrm{nm}$ the molecule can be converted into trans configuration almost quantitatively ( $<3 \%$ cis) (Fig. 6). The photostationary states (PSS) strongly depend on the solvent (Table 2). Dichloromethane and chloroform decrease switching efficiency which could be due to traces of hydrochloric acid. Coordinating solvents improve trans-cis isomerisation. It

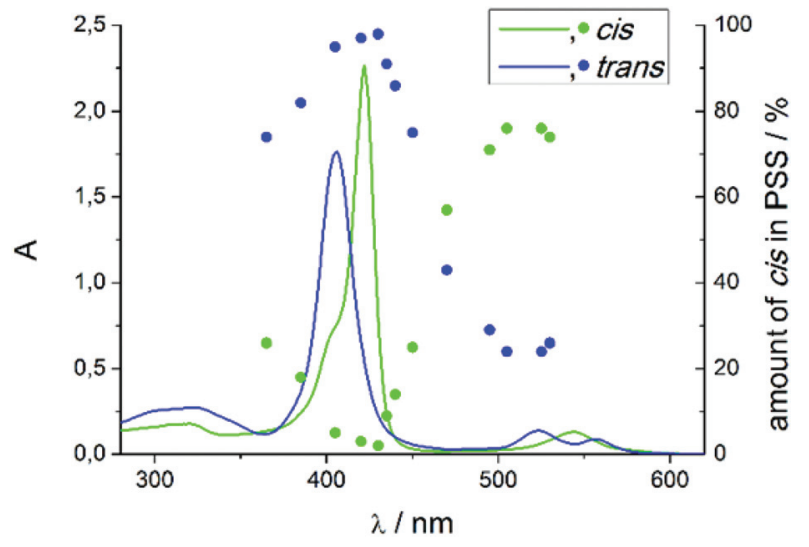

Fig. 6 UV/Vis spectra of trans and cis record player 2 (blue and green line) and the photo steady states (blue and green points) after irradiation with the corresponding wavelength in dimethylsulfoxide (DMSO). The isomers were separated by HPLC.
Table 2 Photostationary states (PSS) of record player 2 in different solvents. Values are determined by integration of corresponding signals in ${ }^{1} \mathrm{H}$ NMR spectra

\begin{tabular}{|c|c|c|}
\hline Solvent & $\begin{array}{l}\text { \% cis } \mathrm{PSS} \\
420 \mathrm{~nm}\end{array}$ & $\begin{array}{l}\% \text { cis } \text { PSS } \\
495 \mathrm{~nm}\end{array}$ \\
\hline Acetone- $\mathrm{d}_{6}$ & $<5$ & 61 \\
\hline Acetonitrile- $\mathrm{d}_{3}$ & $<5$ & 65 \\
\hline Benzene- $\mathrm{d}_{6}$ & $<5$ & 63 \\
\hline Chloroform-d & $<5$ & 18 \\
\hline Cyclohexane- $\mathrm{d}_{12}$ & $<5$ & 59 \\
\hline Dichloromethane- $\mathrm{d}_{2}$ & $<5$ & 45 \\
\hline DMSO-d 6 & $<5$ & 75 \\
\hline Methanol-d ${ }_{4}$ & $<5$ & 69 \\
\hline Pentafluoro benzonitrile & $<5$ & 67 \\
\hline Tetrachloromethane & $<5$ & 57 \\
\hline Tetrahydrofuran- $\mathrm{d}_{8}$ & $<5$ & 75 \\
\hline Toluene- $\mathrm{d}_{8}$ & $<5$ & 65 \\
\hline
\end{tabular}

seems that a solvent molecule stabilizes the paramagnetic cis isomer by formation of the six fold coordinated complex.

This stabilisation also affects the thermal half-life of the cis form. In coordinating solvents the cis isomer is highly stable. The half-life in DMSO is about 400 days $\left(20^{\circ} \mathrm{C}\right)$, which, to our knowledge, is the highest value ever observed for an azo compound. In non-coordinating solvents it is much shorter (dichloromethane: $63 \mathrm{~d}\left(20^{\circ} \mathrm{C}\right)$, chloroform: $29 \mathrm{~d}\left(20^{\circ} \mathrm{C}\right)$ ), but still longer than for usual azo compounds. ${ }^{39,40}$ The photo physics of the isomerisation is very unusual and not yet understood. Particularly the energy transfer from the porphyrin to the azopyridine unit is unclear. Mechanistic investigations on metal free and $\mathrm{Zn}$ containing record player molecules are under way. The fact that the meso phenyl substituted nickel record player (1) has poor switching properties (Fig. 7) leads to the conclusion that the isomerisation is strongly coupled to the spin state switch.

This pertains to both directions. Irradiation into the $\mathrm{Q}$ band (495-530 nm) will only enrich the cis isomer if coordination takes place, and irradiation into the Soret band (405-435 nm) will only convert the molecule to its trans configuration if a decoordination occurs. Upon irradiation with a shorter wavelength $(365 \mathrm{~nm})$ the photochromic behaviors of $\mathbf{1}$ and 2 become quite similar and independent of the spin state switch. In this region the $\pi \pi^{*}$ band of the azopyridine is located. So the spin state switch seems only to be crucial for

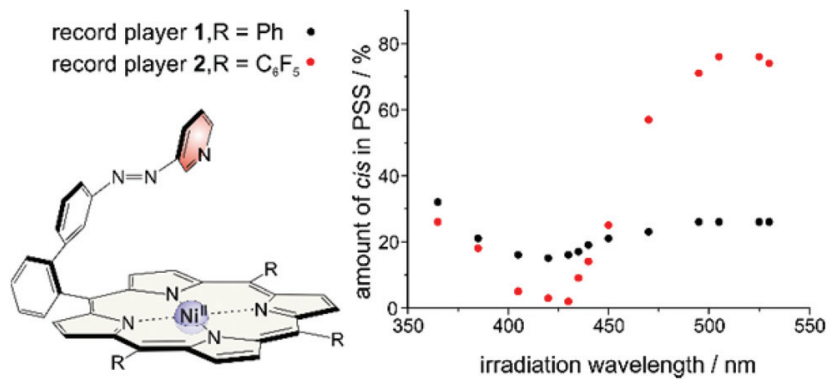

Fig. 7 Switching properties of meso phenyl (1) and meso pentafluorophenyl (2) substituted record players. 

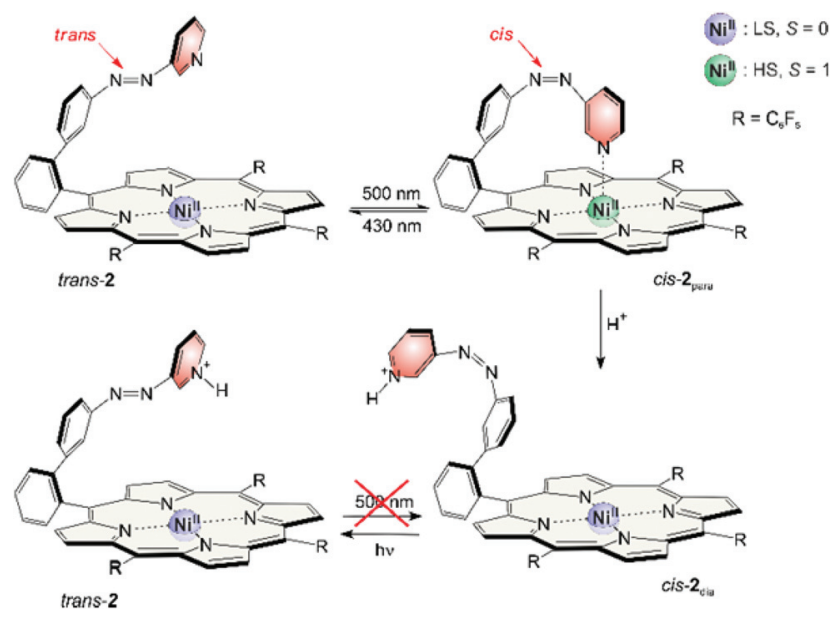

Fig. 8 Photo switching between trans-2 and cis-2 para was achieved with 500 and $430 \mathrm{~nm}$ (top). After conversion into the respective protonated forms, photo conversion is only possible from the cis to the trans configuration (bottom).

Table 3 Record player 2 as a molecular digital logic gate (truth table)

\begin{tabular}{lll}
\hline $\begin{array}{ll}\text { Input } \\
h \nu\end{array}$ & $\begin{array}{l}\text { Input } \\
\mathrm{pH}\end{array}$ & $\begin{array}{l}\text { Output } \\
h \nu \& \mathrm{pH}\end{array}$ \\
\hline $500 \mathrm{~nm}$ & $\mathrm{OH}^{-}$ & para \\
$500 \mathrm{~nm}$ & $\mathrm{H}^{+}$ & dia \\
$430 \mathrm{~nm}$ & $\mathrm{OH}^{-}$ & dia \\
$430 \mathrm{~nm}$ & $\mathrm{H}^{+}$ & dia
\end{tabular}

the isomerisation if it is indirectly induced by irradiation into a porphyrin absorption band (Soret or Q band) (Fig. 8).

Magnetic states and photo switching can be manipulated by changing the $\mathrm{pH}$ value. Addition of acids ( $<1 \% \mathrm{TFA})$ converts cis-2 to a protonated form which is completely diamagnetic. Photo induced back-isomerisation is possible with any wavelength. Protonated trans-2 does not isomerise to the cis configuration (e.g. by irradiation into the $\mathrm{Q}$ band).

Assuming light $(430 / 500 \mathrm{~nm})$ and $\mathrm{pH}$ (high/low) as input and the magnetic state (dia-/paramagnetic) as output, record player 2 can viewed as a molecular logic gate (truth table see Table 3). The molecule is extremely robust (several thousand switching cycles), and the spin state allows a nondestructive readout. Assuming $500 \mathrm{~nm}$ light, $\mathrm{pH}$ high and paramagnetic spin state as digital 1 the molecule corresponds to an AND gate.

\subsection{Methods for magnetic measurements}

Beside the photochromism the magnetic properties are the second important (and probably the most interesting) features of the record player 2. The LD-CISSS approach is based on the assumption that the nickel complex is completely diamagnetic in trans and completely paramagnetic in cis configuration. However, one can essentially assume that the trans isomer should exhibit at least some residual paramagnetism because of intermolecular coordination, and the cis form might not be $100 \%$ paramagnetic because of incomplete binding of the azo-

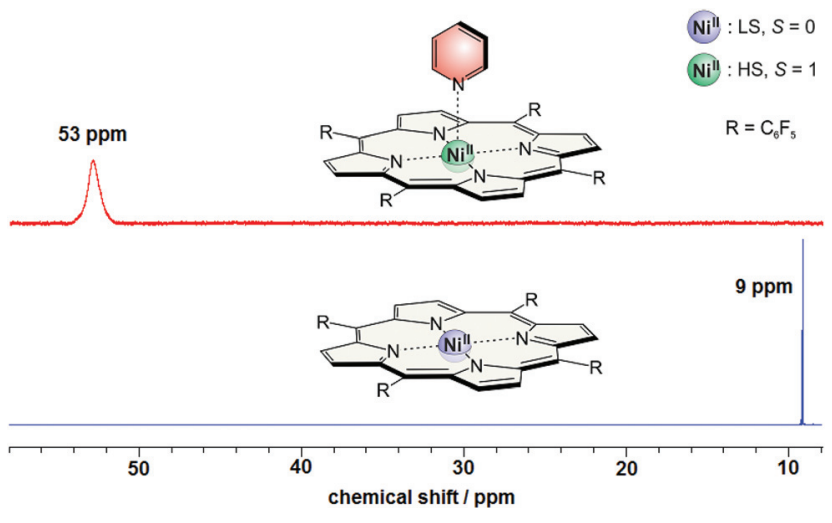

Fig. $9{ }^{1} \mathrm{H}$ NMR spectra of Ni-TPPF 20 with (red) and without (blue) pyridine $(500 \mathrm{MHz}$, acetonitrile, $300 \mathrm{~K})$.

pyridine arm. To quantify the paramagnetism of a Ni-porphyrin the chemical shifts of the pyrrole protons in ${ }^{1} \mathrm{H}$ NMR spectra were investigated. Diamagnetic Ni-TPPF 20 gives rise to a shift of about $8.9(+/-0.4) \mathrm{ppm}$ depending on the solvent while the proton signals of a paramagnetic complex appear at $53(+/-0.4) \mathrm{ppm}$. This fact is shown in Fig. 9. By addition of pyridine a paramagnetic complex is formed which leads to a downfield shift of $44 \mathrm{ppm}$ (from 8.9 to $53 \mathrm{ppm}$ ). The maximum shift only depends on the solvent $(+/-0.4 \mathrm{ppm})$ but not on the nature of the axial ligand (see ESI $\dagger$ ). The magnetic moment of the high-spin species (2.9 B.M.) was confirmed by Evans measurements. ${ }^{33,41}$

Because of the fast ligand exchange a time average of the pyrrole signals of dia- and paramagnetic Ni-porphyrin molecules is observed. The chemical shift $\delta$ is a linear function of the mole fraction of paramagnetic nickel (amount of paramagnetic nickel divided by the total amount of nickel):

$$
\chi_{\text {para }}=\frac{\delta-\delta_{\text {dia }}}{\delta_{\text {para }}-\delta_{\text {dia }}}
$$

$\chi_{\text {para }}$, mole fraction of paramagnetic Ni-porphyrin; $\delta_{\text {para }}$, paramagnetic shift of pyrrole protons (53 ppm); $\delta_{\text {dia }}$, diamagnetic shift of pyrrole protons (8.9 ppm); $\delta$, observed shift of pyrrole protons [ppm].

There is formation of square pyramidal complexes as well. However, square pyramidal and square bipyramidal complexes exhibit approximately the same paramagnetic shifts which can be shown by titration experiments (see ESI†).

\subsection{Intermolecular coordination}

Dilution series of trans-2 show a concentration dependence of the chemical shifts of the pyrrole signals (Fig. 10). A concentrated solution gives rise to a slightly downfield shift indicating paramagnetism due to intermolecular coordination. The magnitude of this effect strongly depends on the solvent. Polar (coordinating) solvents like acetonitrile and DMSO suppress intermolecular coordination while nonpolar solvents like cyclohexane have no effect. There is a linear correlation between the average paramagnetic shift $\left(\delta_{\text {para }}\right)$ and the concen- 

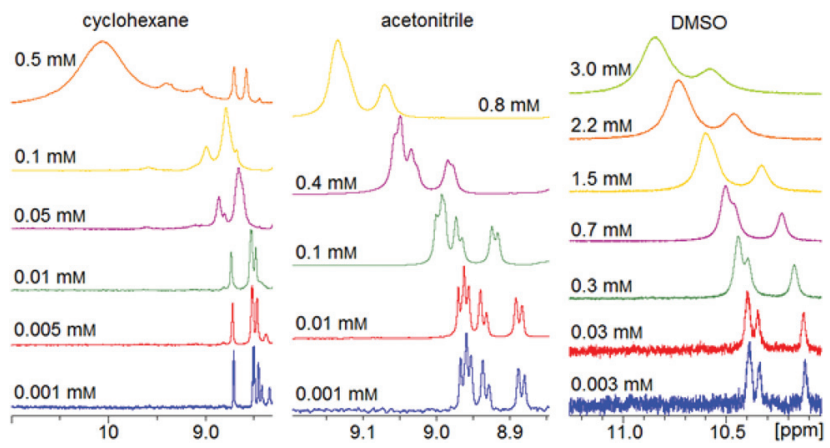

Fig. $10{ }^{1} \mathrm{H}$ NMR spectra of record player 2 in trans configuration in different solvents $(500 \mathrm{MHz}, 300 \mathrm{~K})$. The downfield shift and signal broadening indicates intermolecular coordination.
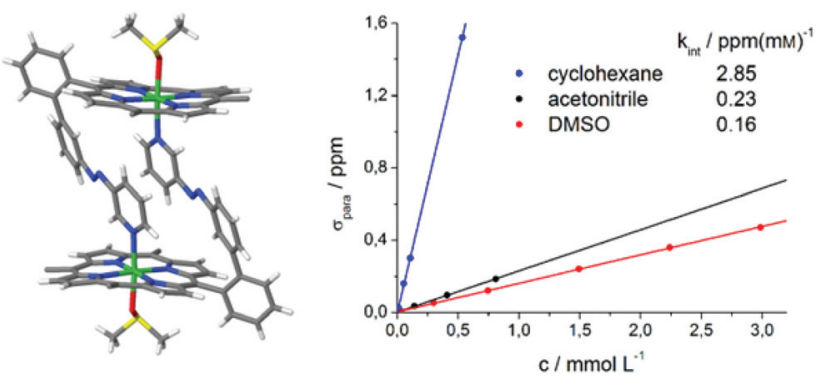

Fig. 11 Crystal structure of trans-2 shows formation of dimers and a sixfold coordination sphere of $\mathrm{Ni}^{2+}$ complemented by one axial DMSO molecule (left). The pentafluorophenyl groups at the porphyrin rings are omitted for clarity. For details see ESI Fig. S4. $\dagger$ The linear correlation between paramagnetic shift of pyrrole signals $\left(\delta_{\text {para }}\right)$ and concentration of trans-2 (right) confirms that there is also intermolecular coordination in solution.

tration (Fig. 11). The slope $\left(k_{\mathrm{int}}\right)$ is a solvent specific parameter representing the tendency for the formation of paramagnetic aggregates.

The crystal structure of trans-2 (crystallized from DMSO) provides information for the mode of interaction, and the strong solvent dependence of intermolecular coordination. trans-2 forms head to tail dimers (Fig. 11). The remaining axial coordination sites are complemented with DMSO. We interpret the reduced intermolecular coordination in DMSO, acetonitrile, methanol, and THF by competition of dimer (or oligomer) formation and coordination by the solvent. For most applications a low degree of intermolecular aggregation (small $k_{\text {int }}$, Fig. 13) is advantegeous. For a efficient LD-CISSS the trans configuration should be as diamagnetic as possible in solution. From the $k_{\text {int }}$ value for cyclohexane $\left(2.85 \mathrm{ppm} \mathrm{mM}^{-1}\right)$ a mole fraction of $6.5 \%$ paramagnetic species in a $1 \mathrm{mM}$ solution due to intermolecular coordination can be derived. In DMSO $(0.4 \%)$ this effect is negligible.

\subsection{Intramolecular coordination}

NMR signals of cis record player 2 do not show any concentration dependence. But we found that pyrrole signals have

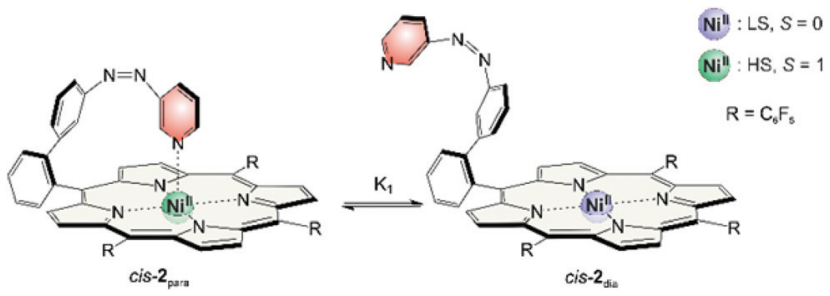

Fig. 12 Equilibrium of the magnetic conformers cis-2 $\mathbf{2}_{\text {para }}$ (left) and cis-2 $2_{\text {dia }}$ (right).

Table 4 Mole fraction of paramagnetic cis-2 (\% cis- $\left.2_{\text {para }}\right)$ relative to the total amount of cis-2 (equilibrium see Fig. 12), calculated from the average ${ }^{1} \mathrm{H}$ NMR chemical shifts of the pyrrole protons

\begin{tabular}{lll}
\hline Solvent & $\begin{array}{l}\text { Shift of pyrrole } \\
\text { proton/ppm }\end{array}$ & $\begin{array}{l}\% \\
\text { cis-2 } 2 \text { para }\end{array}$ \\
\hline Acetone & 42 & 75 \\
Acetonitrile & 44 & 80 \\
Benzene & 46 & 84 \\
Cyclohexane & 46 & 84 \\
Dichloromethane & 37 & 64 \\
DMSO & 49 & 91 \\
Methanol & 47 & 86 \\
Pentafluoro benzonitrile & 45 & 82 \\
Tetrachloromethane & 43 & 77 \\
Tetrahydrofuran & 50 & 93 \\
Toluene & 46 & 84
\end{tabular}

different chemical shifts depending on the solvent (Table 4). The maximum shift $\delta_{\max }$ (eqn (1)) of $53 \mathrm{ppm}$ was confirmed by addition of pyridine- $\mathrm{d}_{5}$. However, in non-coordinating solvents such as cyclohexane or benzene the chemical shift of the pyrrole protons is lower (46 ppm) which indicates that the cis isomer is not completely paramagnetic. Obviously there is a fast equilibrium between the intramolecularly coordinated paramagnetic species ( cis-2 $_{\text {para }}$ ) and a non-coordinated diamagnetic form (cis-2 $\left.\mathrm{2}_{\mathrm{dia}}\right)$. The two species are magnetic conformers (Fig. 12).

In DMSO and tetrahydrofuran the proportions of cis-2 $\mathbf{2}_{\text {para }}$ (91 and 93\%) are quite close to the maximum value. The properties of these polar solvents obviously favor the paramagnetic conformer. Previous studies on the association constants of axial ligands to Ni-porphyrins could give a hint why this is the case. Binding of the first axial ligand $\left(K_{1}\right)$ is less exergonic than association of the second $\left(K_{2}\right)$ (except for very strong ligands). DMSO and THF are not sufficiently strong ligands to efficiently bind to the square planar Ni-porphyrin ( $K_{1}$ is small). However, intramolecular coordination of the azopyridine in the cis-isomer activates the sixth binding site sufficiently to bind a weak ligand such as DMSO. ${ }^{33}$ Hence, upon addition of a solvent molecule the square bipyramidal complex is formed $\left(K_{2}\right)$ which is now stabilized and which cannot directly convert into its diamagnetic form. Note that DMSO also complements the sixth binding site in the X-ray structure of trans-2. It is striking that the same solvents giving rise to a large downfield pyrrole shift in cis-2 also favor the conversion of trans-2 to cis-2 upon irradiation at $495 \mathrm{~nm}$ (see Table 2). 


\subsection{Temperature dependence of NMR shifts}

The high upfield shift of NMR signals of the paramagnetic complex is mainly due to the hyperfine contact shift. ${ }^{42-44}$ Isotropic shifts $\left(\delta_{\text {para }}=\delta_{\text {obs }}-\delta_{\text {dia }}\right)$ can be determined accurately comparing cis-2 with the corresponding diamagnetic zinc derivative. Temperature dependence of chemical shifts show Curie behavior with a reasonable good fit. The slopes correlate with the magnitude of the hyperfine coupling. For the ortho pyridine protons the interaction is strongest. At $300 \mathrm{~K}$ the signals are already at about $100 \mathrm{ppm}$. Signals vanish at lower temperatures because they become too broad, and relaxation is too fast. All other shifts are shown in Fig. 13. Some intercepts differ significantly from Curie law which can be explained by the presence of a second spin species namely the diamagnetic cis-2 $2_{\text {dia. }}$. This seems reasonable taking into account that measurements are performed in dichloromethane in which the amount of diamagnetic cis isomer is high (36\%).

From temperature dependent NMR measurements thermodynamic parameters $\Delta H$ and $\Delta S$ can be determined as well. The intramolecular association constant $K_{1}$ (see Fig. 12) can be calculated directly from the observed pyrrole proton shifts in ${ }^{1} \mathrm{H}$ NMR spectra (ESI $\dagger$ ). Temperature dependence of $K_{1}$ gives $\Delta H$ and $\Delta S$ by the Gibbs free enthalpy relation. The experimental values are in good agreement with the calculated (B3LYP/def2TZVP//PBE/SVP) energies which again confirms

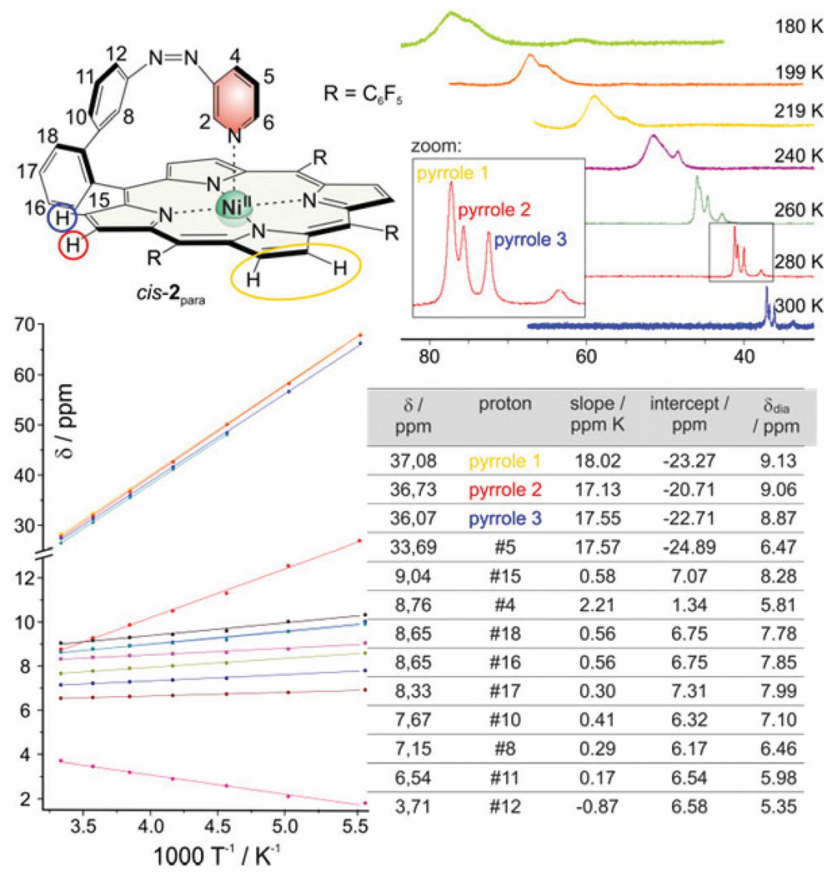

Fig. 13 Temperature dependence of chemical shifts of cis-2 in ${ }^{1} \mathrm{H}$ NMR spectra $\left(500 \mathrm{MHz}\right.$, dichloromethane- $\left.\mathrm{d}_{2}\right)$. Shifts of the pyrrole protons as a function of temperature are shown top right. The table bottom right shows the chemical shifts of all protons (except those of ortho pyridine $\# 2$ and \#6) at $300 \mathrm{~K}(\delta)$, the slopes and intercepts of the corresponding Curie plots (bottom left) and the diamagnetic shifts of the corresponding zinc derivative $\left(\delta_{\text {dia }}\right){ }^{18}$
Table 5 Association constants $K_{1}(300 \mathrm{~K})$, experimental (exp., DMSO$\mathrm{d}_{6}$ ) and calculated (calc., B3LYP/def2TZVP//PBE/SVP) and thermodynamic parameters $\Delta H\left(\mathrm{kcal} \mathrm{mol}^{-1}\right)$ and $\Delta S\left(\mathrm{cal} \mathrm{mol}^{-1} \mathrm{~T}^{-1}\right)$ for the intramolecular coordination of cis-1 and cis-2 (Fig. 12)

\begin{tabular}{lll}
\hline & cis-1 & $c i s-2$ \\
\hline$K_{1}(300 \mathrm{~K})$ & 0.0616 & 7.4674 \\
$\Delta H$ (exp.) & $-1.19( \pm 0.09)$ & $-3.95( \pm 0.13)$ \\
$\Delta H$ (calc.) & $-0.25( \pm 0.28)$ & -3.26 \\
$\Delta S$ (exp.) & $-9.52( \pm 0.43)$ \\
$\Delta G_{300 \text { к (exp.) }}$ & $1.67( \pm 0.17)$ & $-9.18( \pm 0.26)$
\end{tabular}

that the used level of theory is suitable for the investigated complexes (Table 5).

The values for the association constant $(7.47$ at $300 \mathrm{~K})$, enthalpy $\left(-3.95 \mathrm{kcal} \mathrm{mol}^{-1}\right)$ and entropy $\left(-9.18 \mathrm{cal} \mathrm{mol}^{-1} \mathrm{~T}^{-1}\right)$ of cis-2 are in good agreement with values obtained for free pyridine and azopyridine ligands. ${ }^{22-24}$ Binding enthalpy is smaller compared to pyridines which is compensated by a smaller entropy. As expected the binding enthalpy for the meso phenyl substituted record player $\mathbf{1}$ is much lower $(-1.19 \mathrm{kcal}$ $\mathrm{mol}^{-1}$ ) whereas entropy is almost equal $\left(-9.52 \mathrm{cal} \mathrm{mol}^{-1} \mathrm{~K}^{-1}\right)$. The thermodynamic data above explain why the phenyl substituted record player cis-1 is not paramagnetic. Even though the geometry is suitable for coordination the binding is endergonic $\left(\Delta G=1.67 \mathrm{kcal} \mathrm{mol}^{-1}\right)$. Coordination of the azopyridine "tonearm" in electron deficient cis-2, however, is clearly exergo$\operatorname{nic}\left(\Delta G=-1.20 \mathrm{kcal} \mathrm{mol}^{-1}\right)$.

\subsection{Structure and molecular dynamics}

Record player cis-2 $\mathbf{2}_{\text {para }}$ does not exhibit symmetry $\left(C_{1}\right)$. However, the NMR spectra are rather in agreement with a time averaged $C_{\mathrm{s}}$ symmetry. The fact that there are only three pyrrole proton signals in cis-2 (Fig. 10 and 13) can be explained by the fast conversion of the two respective enantiomers. The ${ }^{19} \mathrm{~F}$ NMR spectra add more evidence for this process. cis-2 exhibits four signals for ortho fluorine $(1: 2: 2: 1)$, two signals for para fluorine $(1: 2)$ and three signals for meta fluorine $(1: 2: 3)$ atoms (Fig. 14).

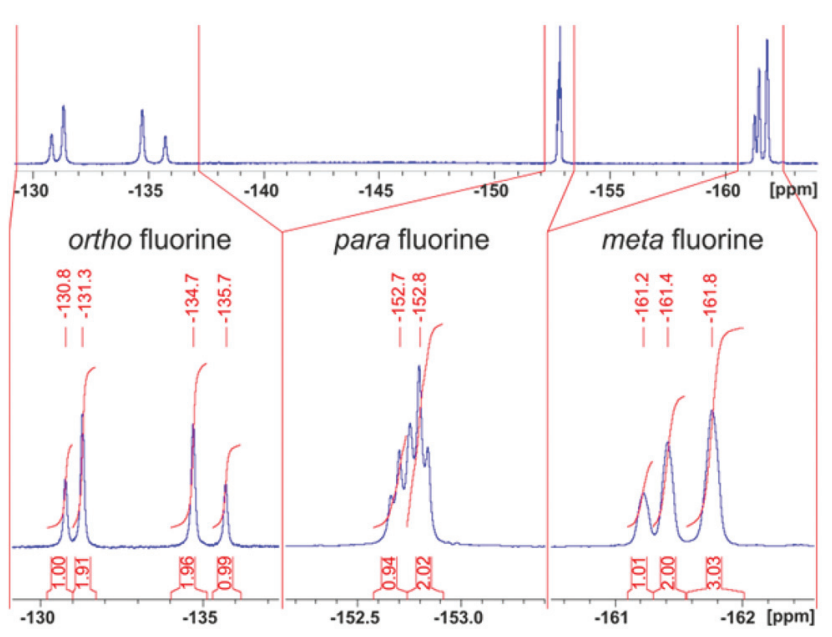

Fig. $14{ }^{19} \mathrm{~F}-\mathrm{NMR}$ spectra of cis-2 (500 MHz, dichloromethane, $\left.300 \mathrm{~K}\right)$. 

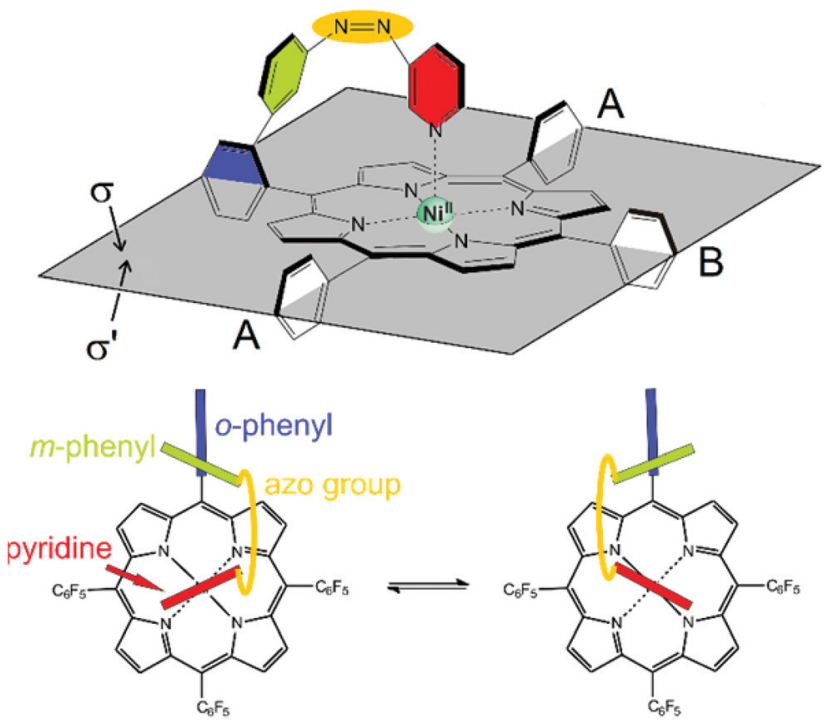

Fig. 15 Top: Record player cis- $\mathbf{2}_{\text {para }}$ has two symmetry non-equivalent sides $\sigma$ and $\sigma^{\prime}$. Neither of the meso substituents (pentafluorophenyl and "tone arm") rotate around the porphyrin plane on the NMR time scale at $300 \mathrm{~K}$. For the sake of clarity the fluorine atoms at the meso positions A and B are omitted. Bottom: top view on the conversion between the two enantiomers of cis-2. The conformational equilibrium is fast on the NMR time scale at $300 \mathrm{~K}$.

Information about the dynamic behavior of cis-2 can be derived from the signal pattern. Since more than three ortho fluorine signals are observed the rotation of the pentafluorophenyl groups in meso position of the porphyrin must be hindered. Hence the molecule has two sides $\left(\sigma\right.$ and $\left.\sigma^{\prime}\right)$ with chemical non-equivalent fluorine atoms. cis-2 as shown in Fig. 12 has $C_{1}$ symmetry which should exhibit six ortho fluorine signals. The fact that there are only four signals and that the ratio given by the integrals is $1: 2: 2: 1$ indicates that the two meso substituents A must be equal. (Fig. 15) This is the case if cis-1 is in equilibrium with its enantiomer and conversion is faster than NMR time scale. The conversion is only a reorientation to a different conformation and therefore should not need much activation energy (Fig. 15). However, racemisation can only proceed via a (short-lived) decoordinated (diamagnetic) state. ${ }^{19} \mathrm{~F}$ signals of meta and para fluorine are in agreement with this hypothesis.

\section{Conclusions}

We present the rational design of a molecule whose spin state can be switched with visible light at room temperature in homogenous solution. Our approach is based on a three-part system: a photoswitchable azopyridine unit, a Ni-porphyrin, and a tether that connects the latter two building blocks ("record player" or "photodissociable ligand" design). Upon irradiation the azopyridine reversibly changes configuration which leads to coordination/decoordination of the pyridine to the $\mathrm{Ni}$ ion. The change of coordination number in turn switches the spin state of the $\mathrm{Ni}^{2+}$ between low-spin (diamagnetic) and high-spin (paramagnetic). We coined our approach light-driven coordination-induced spin state switching (LD-CISSS). To find a system with high switching efficiency, a number of systems differing in tether structure, and position of attachment were evaluated in silico by DFT calculations. Among 36 candidates three structures were identified as potentially operative, and worthwhile being synthesized. One of the three structures was actually prepared, and thoroughly investigated. While the basic properties have been published recently, ${ }^{18}$ we here present a detailed experimental and computational study of the system. DFT calculations and experiments reveal that both, geometry and electronic requirements have to be accurately met to obtain an efficient system. The tether has to be constructed in such a way that the pyridine nitrogen lone pair is placed exactly perpendicular to the porphyrin plane, and at a distance of 2.25 to $2.35 \AA$. Moreover, the Ni-porphyrin must be electron poor. The "record player" compound with phenyl substituents at the meso positions exhibits no spin state switching, whereas the corresponding system with three pentafluorophenyl substituents is highly efficient. According to DFT calculations, and corresponding NMR experiments, electron withdrawing substituents at the porphyrin meso positions strongly improve the coordination of the pyridine to the $\mathrm{Ni}$ ion, which is a prerequisite for the spin change. Switching efficiency also strongly depends on the solvent. Weakly coordinating solvents prevent intermolecular coordination, and concomitantly improve intramolecular binding. The mole fraction of paramagnetic cis-2 in DMSO is $91 \%$ and $93 \%$ in THF. Thermal isomerisation of the cis isomer back to the thermodynamically more stable trans form is slowed down considerably as well in weakly coordinating solvents. Thermal half-life of the high-spin cis isomer in DMSO is extraordinarily long (400 days at $20^{\circ} \mathrm{C}$ ). So DMSO seems to be the solvent of choice. Considering the extreme long-term switching stability (more than 20000 cycles with no detectable side products) and the fact that it can be switched with visible light, combined with the above mentioned properties, "record player" 2 is superior to conventional azobenzenes in most aspects. Spin state switching is inhibited at low pH. If light (500/ $430 \mathrm{~nm}$ ) and $\mathrm{pH}$ (low/high) is used as input, and the magnetic state (dia/paramagnetic) as output the molecule can be used as a logic gate (AND gate). The fact that the magnetic state can be used as output has the advantage that it provides means for a non-destructive readout (orthogonal to light and $\mathrm{pH})$.

Room temperature switchable molecular magnets provide the potential for a number of interesting applications such as information storage ${ }^{45,46}$ and processing, ${ }^{47}$ sensor applications, ${ }^{48}$ switchable contrast agents for $\mathrm{MRI}^{49-51}$ or light induced magnetic levitation. ${ }^{41}$ Further improvement based on the detailed information provided above should be possible. However, the mechanism of the unusual energy transfer from the porphyrin unit to the azopyridine ligand has yet to be elucidated. 


\section{Acknowledgements}

This work has been supported by the Deutsche Forschungsgemeinschaft within the Sonderforschungsbereich 677 "Function by Switching".

\section{Notes and references}

1 M. P. Shores, C. M. Klug and S. R. Fiedler, Spin-Crossover Materials: Properties and Applications, ed. M. A. Halcrow, John Wiley \& Sons, Ltd., 1st edn, 2013, ch. 10, pp. 281-301.

2 C. Gandolfi, G. G. Morgan and M. Albrecht, Dalton Trans., 2012, 41, 3726-3730.

3 P. N. Martinho, Y. Ortin, B. Gildea, C. Gandolfi, G. McKerr, B. O'Hagan, M. Albrecht and G. G. Morgan, Dalton Trans., 2012, 41, 7461-7463.

4 M. P. Shores, C. M. Klug and S. R. Fiedler, Spin-Crossover Materials, ed. M. A. Halcrow, 2013, pp. 281-301.

5 R. N. Muller, L. Vander Elst and S. Laurent, J. Am. Chem. Soc., 2003, 125, 8405-8407.

6 C. Rajadurai, M. Ruben and D. Kruk, EP 2072062 A1, 2009.

7 G. M. Clore and J. Iwahara, Chem. Rev., 2009, 109, 41084139.

8 A. R. Rocha, V. M. Garcia-suarez, S. W. Bailey, C. J. Lambert, J. Ferrer and S. Sanvito, Nat. Mater., 2005, 4, 335-339.

9 K. Hamachi, K. Matsuda, T. Itoh and H. Iwamura, Bull. Chem. Soc. Jpn., 1998, 71, 2937.

10 M. M. Paquette, B. O. Patrick and N. L. Frank, J. Am. Chem. Soc., 2011, 133, 10081-10093.

11 A. Bannwarth, S. O. Schmidt, G. Peters, F. D. Sönnichsen, W. Thimm, R. Herges and F. Tuczek, Eur. J. Inorg. Chem., 2012, 2012, 2776-2783.

12 Y. Hasegawa, S. Kume and H. Nishihara, Dalton Trans., 2009, 280-284.

13 R. K. Wilson and S. Brooker, Dalton Trans., 2013, 42, 12075-12078.

14 N. Tanifuji, M. Irie and K. Matsuda, J. Am. Chem. Soc., 2005, 127, 13344-13353.

15 X. Ge, C. Manzano, R. Berndt, L. T. Anger, F. Köhler and R. Herges, J. Am. Chem. Soc., 2009, 131, 6096-6098.

16 T. G. Gopakumar, F. Matino, H. Naggert, A. Bannwarth, F. Tuczek and R. Berndt, Angew. Chem., Int. Ed., 2012, 51, 6262-6266.

17 T. G. Gopakumar, M. Bernien, H. Naggert, F. Matino, C. F. Hermanns, A. Bannwarth, S. Mühlenberend, A. Krüger, D. Krüger, F. Nickel, W. Walter, R. Berndt, W. Kuch and F. Tuczek, Chem. - Eur. J., 2013, 19, 1570215709.

18 S. Venkataramani, U. Jana, M. Dommaschk, F. D. Sönnichsen, F. Tuczek and R. Herges, Science, 2011, 331, 445-448.

19 D. Achey and G. J. Meyer, Inorg. Chem., 2013, 52, 9574-9582.

20 T. J. Lotz and T. A. Kaden, J. Chem. Soc., Chem. Commun., 1977, 15-16.
21 Y. Song, R. E. Haddad, S.-L. Jia, S. Hok, M. M. Olmstead, D. J. Nurco, N. E. Schore, J. Zhang, J.-G. Ma, K. M. Smith, S. Gazeau, J. Pécaut, J.-C. Marchon, C. J. Medforth and J. A. Shelnutt, J. Am. Chem. Soc., 2005, 127, 11791192.

22 S. Thies, C. Bornholdt, F. Köhler, F. D. Sönnichsen, C. Näther, F. Tuczek and R. Herges, Chem. - Eur. J., 2010, 16, 10074-10083.

23 S. Thies, H. Sell, C. Bornholdt, C. Schütt, F. Köhler, F. Tuczek and R. Herges, Chem. - Eur. J., 2012, 18, 1635816368.

24 S. Thies, H. Sell, C. Schütt, C. Bornholdt, C. Näther, F. Tuczek and R. Herges, J. Am. Chem. Soc., 2011, 133, 16243-16250.

25 H. K. Hombrecher and K. Lüdtke, Tetrahedron, 1993, 49, 9489-9494.

26 K. H. Neumann and F. Vogtle, J. Chem. Soc., Chem. Commun., 1988, 520-522.

27 C. A. Hunter and L. D. Sarson, Tetrahedron Lett., 1996, 37, 699-702.

28 H. Sugimoto, K. Kuramoto and S. Inoue, J. Chem. Soc., Perkin Trans. 1, 2002, 1826-1830.

29 L. J. Esdaile, P. Jensen, J. C. McMurtrie and D. P. Arnold, Angew. Chem., Int. Ed., 2007, 46, 2090-2093.

30 M. V. Peters, R. Goddard and S. Hecht, J. Org. Chem., 2006, 71, 7846-7849.

31 A. M. G. Silva, A. C. Tom'e, M. G. P. M. S. Neves, A. M. S. Silva and J. A. S. Cavaleiro, J. Org. Chem., 2005, 70, 2306-2314.

32 A. M. G. Silva, P. S. S. Lacerda, A. C. Tom'e, M. G. P. M. S. Neves, A. M. S. Silva, J. A. S. Cavaleiro, E. A. Makarova and E. A. Lukyanets, J. Org. Chem., 2006, 71, 8352-8356.

33 F. Matino, G. Schull, U. Jana, F. Köhler, R. Berndt and R. Herges, Chem. Commun., 2010, 46, 6780-6782.

34 A. A. Starikova, R. M. Minyaev, A. G. Starikov and V. I. Minkin, Eur. J. Inorg. Chem., 2013, 2013, 4203-4219.

35 D. Kim, Y. O. Su and T. G. Spiro, Inorg. Chem., 1986, 25, 3988-3993.

36 S. Grimme, J. Antony, S. Ehrlich and H. Krieg, J. Chem. Phys., 2010, 132, 154104.

37 F. A. Walker, E. Hui and J. M. Walker, J. Am. Chem. Soc., 1975, 97, 2390-2397.

38 S. J. Cole, G. C. Curthoys, E. A. Magnusson and J. N. Phillips, Inorg. Chem., 1972, 11, 1024-1028.

39 R. J. W. Le Fevre and J. Northcott, J. Chem. Soc., 1953, 867870.

40 E. R. Talaty and J. C. Fargo, Chem. Commun., 1967, 65-66.

41 D. F. Evans, J. Chem. Soc., 1959, 2003-2005.

42 J. A. Happe and R. L. Ward, J. Chem. Phys., 1963, 39, 12111218.

43 C. Belle, C. Bougault, M.-T. Averbuch, A. Durif, J.-L. Pierre, J.-M. Latour and L. Le Pape, J. Am. Chem. Soc., 2001, 123, 8053-8066.

44 I. Bertini, P. Turano and A. J. Vila, Chem. Rev., 1993, 93, 2833-2932. 
45 L. Wei, K. Padmaja, W. J. Youngblood, A. B. Lysenko, J. S. Lindsey and D. F. Bocian, J. Org. Chem., 2003, 69, 1461-1469.

46 K. L. Kompa and R. D. Levine, Proc. Natl. Acad. Sci. U. S. A., 2001, 98, 410-414.

47 K. Szacilowski, Chem. Rev., 2008, 108, 3481-3548.
48 J. M. Perez, L. Josephson, T. O’Loughlin, D. Hogemann and R. Weissleder, Nat. Biotechnol., 2002, 20, 816-820.

49 Christian-Albrechts-Universität and Universitätsklinikum S.-H., WO 2012/022299, patent pending.

50 R. Herges, Nachr. Chem., 2011, 59, 817-821.

51 R. B. Lauffer, Chem. Rev., 1987, 87, 901-927. 